\title{
Kinematics of the ionised gas in the spiral galaxy NGC 2403*
}

\author{
F. Fraternali ${ }^{1,2}$, T. Oosterloo ${ }^{2}$, and R. Sancisi ${ }^{3,4}$ \\ 1 Theoretical Physics, University of Oxford, 1 Keble Road, Oxford OX1 3NP, UK \\ e-mail: filippo@thphys.ox.ac.uk \\ 2 ASTRON, PO Box 2, 7990 AA, Dwingeloo, The Netherlands \\ 3 INAF, Osservatorio Astronomico, via Ranzani 1, 40127, Bologna, Italy \\ 4 Kapteyn Astronomical Institute, University of Groningen, The Netherlands
}

Received 26 March 2004 / Accepted 24 May 2004

\begin{abstract}
We present a study of the kinematics of the ionised gas in the nearby spiral galaxy NGC 2403 using deep long-slit spectra obtained with the 4.2-m William Herschel Telescope. The data show the presence of a halo component of ionised gas that is rotating more slowly than the gas in the disk. The kinematics of this ionised halo gas is similar to that of the neutral halo gas. On small scales, broad line profiles (up to $300 \mathrm{~km} \mathrm{~s}^{-1}$ wide) indicate regions of fast outflows of ionised gas. We discuss these new results in the context of galactic fountain models.
\end{abstract}

Key words. galaxies: individual: NGC 2403 - galaxies: structure - galaxies: kinematics and dynamics - galaxies: halos ISM: kinematics and dynamics

\section{Introduction}

Recent studies of edge-on spiral galaxies have shown the presence of thick gaseous layers extending to large vertical distances from the plane of the disk. Optical observations have revealed extraplanar emission from ionised gas, frequently referred to as DIG (Diffuse Ionised Gas) (Ferguson et al. 1996; Hoopes et al. 1999). Such ionised layers are analogous to the Reynolds' layer of Warm Ionised Medium (WIM) of our Galaxy (Reynolds 1991). They can extend to several kpc above the plane (e.g. $15 \mathrm{kpc}$ in NGC 4631, Donahue et al. 1995; $5 \mathrm{kpc}$ in NGC 5775, Collins et al. 2000), but there are also galaxies that show little extraplanar emission (Rand et al. 1992). Deep H I observations and a detailed modeling of the $\mathrm{H} I$ layers have revealed extraplanar gas also in the neutral phase (NGC 891, Swaters et al. 1997; UGC 7321, Matthews \& Wood 2003). Such extraplanar HI is detected at several $\mathrm{kpc}$ from the plane, up to $10-15 \mathrm{kpc}$, in NGC 891 (Fraternali et al. 2003).

The extraplanar gas has peculiar kinematics that has been studied both for the neutral and the ionised component. The modeling of the HI layer in the edge-on galaxy NGC 891 (Swaters et al. 1997; Fraternali et al. 2003), has shown that the extraplanar gas is not co-rotating with the gas in the plane. Instead, it rotates more slowly with a rotation velocity gradient

* Based on observations made with the WHT operated on the island of La Palma by the Isaac Newton Group in the Spanish Observatorio del Roque de los Muchachos of the Instituto de Astrofisica de Canarias. in the vertical direction of about $10-20 \mathrm{~km} \mathrm{~s}^{-1}$ per kpc and with larger gradients in the inner regions of the galaxy. Indications for such a rotation velocity gradient have also been reported for the super-thin galaxy UGC 7321 (Matthews \& Wood 2003). Long-slit spectroscopic observations of edge-on spiral galaxies show similar results for the ionised component (NGC 891, Rand 1997; NGC 5775, Rand 2000). The velocity gradient seems particularly remarkable in the edge-on galaxy NGC 5775 where the rotation velocity, measured in different emission lines, seems to drop down to almost zero at a distance of $\sim 5-10 \mathrm{kpc}$ from the plane.

The difference in rotation velocity between the planar and the extraplanar gas has been the key factor for revealing the latter also in galaxies viewed at different inclination angles than edge-on. Indeed, in such systems one can separate the "lagging" extraplanar gas kinematically rather than spatially. NGC 2403 is the first galaxy that has been studied for this purpose. It is a nearby Sc spiral very similar to M 33 and viewed at an inclination angle of $60^{\circ}$. We have adopted a distance of $3.18 \mathrm{Mpc}$ (Madore \& Freedman 1991). NGC 2403 is located in the far outskirts of the M 81 group, at a projected distance of more than $1 \mathrm{Mpc}$ from the centre of the group. The closest object is the dwarf spheroidal DDO 44, at a projected distance of $75 \mathrm{kpc}$, but without a clear physical connection with NGC 2403 (Karachentsev et al. 1999). Recent H I observations of NGC 2403 have revealed the presence of tails in the H I spectra at velocities lower than the rotation in the disk (Fraternali et al. 2002a). Such tails (also referred to as "anomalous gas") have been successfully reproduced by models with a thick layer 
("halo") of neutral gas surrounding the thin disk while the thick layer has a mean rotation velocity about $20-50 \mathrm{~km} \mathrm{~s}^{-1}$ lower than the thin disk (Schaap et al. 2000; Fraternali et al. 2001).

The advantage of studying a galaxy viewed at intermediate inclination is that one can investigate the presence of noncircular (inflow/outflow) motions as well. Indeed, in the case of NGC 2403, in addition to the low rotation velocity, also indications for a large-scale radial inflow (of about $10-20 \mathrm{~km} \mathrm{~s}^{-1}$ ) of the halo HI have been found. This has been obtained by comparing the velocity fields of the disk and of the extraplanar gas (Fraternali et al. 2001). Finally, the H I data of NGC 2403 have also shown several gas complexes at quite anomalous (non-circular) velocities. These complexes have masses of about $10^{6}-10^{7} M_{\odot}$ and velocities that depart from rotation by up to $150 \mathrm{~km} \mathrm{~s}^{-1}$ (300 $\mathrm{km} \mathrm{s}^{-1}$ if the gas is moving vertically). They form a population of high velocity clouds in the halo of NGC 2403 that are probably analogous to some of the HVCs of our Galaxy (Wakker \& Van Woerden 1997).

The origin of the extraplanar gas in spiral galaxies is still poorly understood. One possibility is that it is produced by stellar activity in the disk and can be described by the so-called galactic fountain model (Shapiro \& Field 1976; Bregman 1980). In such a model the gas is thought to flow out of the disk in the halo region mostly as ionised gas. Such gas can move to large heights above the plane (decreasing its rotation velocity) and to large distances from its original radius conserving its angular momentum. Then, after cooling and having become neutral, it is expected to fall back onto the disk, eventually acquiring a radial inflow motion. Therefore, in a galactic fountain process, one expects the early (outflow) stage to be mostly traced by ionised gas and the late (inflow) stage by neutral gas. As mentioned above, in NGC 2403 there are indeed indications of a large-scale radial inflow of neutral gas at a velocity of about $10-20 \mathrm{~km} \mathrm{~s}^{-1}$ which can be ascribed to the late stage of a galactic fountain. However, this result, by itself, is not conclusive for the fountain hypothesis. Inflow is obviously expected also in the case of accretion from the intergalactic medium (Oort 1970). Therefore the study of the kinematics of ionised gas is crucial for understanding the origin of the halo gas.

In this paper we present a study of the kinematics of the ionised gas in NGC 2403 using long slit spectroscopy of strong emission lines including $\mathrm{H} \alpha$ and [N II]. The main goal is to investigate the presence of an ionised counterpart of the lagging halo of neutral gas (Sect. 3.2). In particular, we search for outflows of ionised gas that could match the observed inflow of neutral one in the hypothesis of a galactic fountain (Sects. 3.3 and 4.1). Finally, a comparison between the $\mathrm{H} \mathrm{I}$ and $\mathrm{H} \alpha$ rotation curves is also presented (Sect. 3.1).

\section{Observations and data reduction}

Optical spectra of NGC 2403 were obtained during three nights in January 2001 with the 4.2-m William Herschel Telescope at the Observatory Roque de los Muchachos in La Palma (Canary Islands) using the ISIS dual-beam spectrograph. The weather conditions were generally good and the seeing was $\leq 1$ " during the three nights. The ISIS spectrograph on the WHT has two arms ("blue" and "red") which can be exposed simultaneously. The spectra of the blue arm range from 4500 to $5500 \AA$ and include $\mathrm{H} \beta$ and [OIII] (5007 $\AA$ ). The spectra of the red arm (from 6200 to $6800 \AA$ ) include important emission lines: $\mathrm{H} \alpha(6563 \AA),[\mathrm{N}$ II] $(6583 \AA, 6548 \AA)$ and the [S II] doublet (6716 $\AA, 6731 \AA$ ). The aim of our project is to study the presence of faint wings to the spectral lines; thus, we mainly focus on the strong emission lines $\mathrm{H} \alpha$ and [N II] and use only red arm data. The spatial scale of the red arm is 0.32 pixel $^{-1}$ corresponding to $5 \mathrm{pc}$ at the distance of NGC 2403. For the dispersion axis the scale is $0.4 \AA$ pixel $^{-1}$ corresponding to about $18 \mathrm{~km} \mathrm{~s}^{-1}$ pixel $^{-1}$ for lines in the red arm. The velocity resolution is about $40 \mathrm{~km} \mathrm{~s}^{-1}$ which is four times lower than the velocity resolution of the H I data (Fraternali et al. 2002a). We used a slit of 3.7 arcmin long $\times 1.2$ arcsec wide which we placed at different positions along the major and minor axes of NGC 2403 and obtained 48 2D-spectra (24 for each arm of the instrument). Eight observations were taken along the major and the minor axes of the galaxy with a total of six slit positions as shown in Fig. 1. Each individual exposure was $45 \mathrm{~min}$. The central parts of the galaxy $\left(R<1.8^{\prime} \simeq 1.7 \mathrm{kpc}\right)$ have obtained the longest integrations, ranging from 4.5 to $6.75 \mathrm{~h}$. The other observational parameters are summarized in Table 1.

The standard data reduction (bias subtraction, flat-field division, wavelength and flux calibrations) was carried out with IRAF (Image Reduction and Analysis Facility) (Valdes 1992). After wavelength calibration, small differences $(<2 \AA)$ between the central wavelengths of sky lines in different exposures were corrected by shifting all spectra in the dispersion axis to match representative $\mathrm{OH}$ sky lines taken from the night sky atlas (Osterbrock et al. 1996). Then, the spectra taken at the same position and in the same night were combined to remove cosmic rays. For the flux calibration Feige56, LB1240, and Feige 15 were used. Finally, the continuum emission was subtracted from the combined spectra by fitting a spline function of order 3 every row along the dispersion axis.

The order 3 function was the best compromise between a smooth continuum and low residuals.

The critical step in reducing these spectroscopic data is the subtraction of the sky lines. The presence of two bright sky lines close to $\mathrm{H} \alpha$ and of the two faint lines embedding [N II] (Fig. 2) requires a very careful sky subtraction. Because emission from NGC 2403 is detected along the entire slit, the standard technique of using the object-free regions of the CCD to make an estimate of the sky emission cannot be used. Attempts to subtract the sky emission using separate exposures on empty sky did not give satisfactory results (Fig. 2, bottom panel). This is probably due to weak variations of the (absolute and relative) strength of sky lines during the night. To avoid these effects, we subtracted the sky lines directly from the exposures of NGC 2403. Due to galactic rotation the galaxy emission shifts in velocity along the slit (see $\mathrm{H} \alpha$ and [N II] emission in Fig. 2). This allows us to find regions along the slit where it does not overlap with the sky lines. We used these regions (typically 25 pixels) to extract the sky lines. In the exposure shown in Fig. 2, for example, we used the rows 1-25 for the line left to $\mathrm{H} \alpha$ emission and rows 301-325 for the one on the right. 

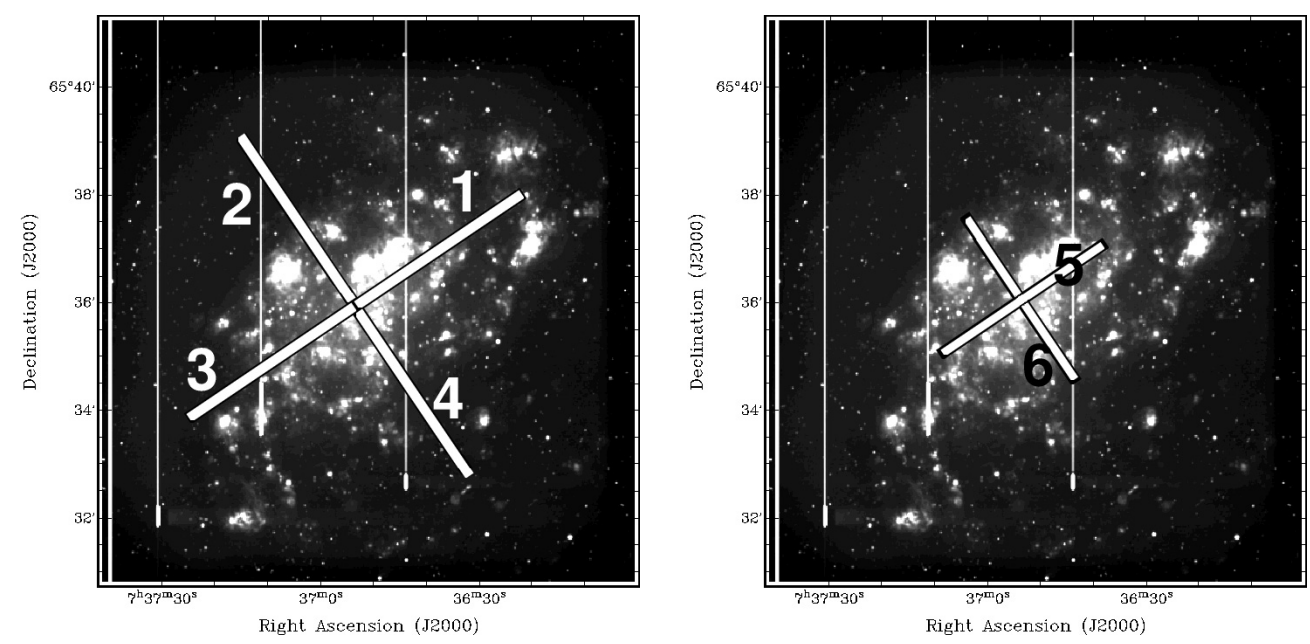

Fig. 1. The 6 long-slits overlaid on an $\mathrm{H} \alpha$ image of NGC 2403, taken from the archive of the Canadian French Hawaiian (CFH) Telescope (Drissen et al. 1999). The total exposure times in each position are given in Table 1.

Table 1. Parameters for the spectroscopic observations of NGC 2403.

\begin{tabular}{ccccccc}
\hline \hline No. & Slit & Location & Date & $\begin{array}{c}\text { Number of } \\
\text { exposures }\end{array}$ & $\begin{array}{c}\text { Exp. time } \\
(\mathrm{h}: \mathrm{min})\end{array}$ & $\begin{array}{c}\text { rms noise } \\
\left(\mathrm{erg} \mathrm{s}^{-1} \mathrm{~cm}^{-2} \AA^{-1} \mathrm{pix}^{-1}\right)\end{array}$ \\
\hline 1 & 1 & major N-W & 2-Jan.-01 & 4 & $3: 00$ & $7.96 \times 10^{-19}$ \\
2 & 2 & minor N-E & 3-Jan.-01 & 2 & $1: 30$ & $1.22 \times 10^{-18}$ \\
3 & 3 & major S-E & 3-Jan.-01 & 5 & $3: 45$ & $7.89 \times 10^{-19}$ \\
4 & 4 & minor S-W & 2-Jan.-01 & 3 & $2: 15$ & $8.28 \times 10^{-19}$ \\
5 & & minor S-W & 3-Jan.-01 & 2 & $1: 30$ & $1.35 \times 10^{-18}$ \\
6 & 5 & major centred & 4-Jan.-01 & 2 & $1: 30$ & $1.67 \times 10^{-18}$ \\
7 & & major centred & 4-Jan.-01 & 2 & $1: 30$ & $1.42 \times 10^{-18}$ \\
8 & 6 & minor centred & 4-Jan.-01 & 4 & $3: 00$ & $1.19 \times 10^{-18}$ \\
\hline
\end{tabular}

We took the mean of these rows and fitted a Gaussian function to the line.

The intensity and the width of sky lines slightly vary along the spatial axis. In order to take this effect into account we have fitted Gaussian functions to other sky lines (e.g. the bright line at about $6579 \AA$ ) in the same spatial regions (1-25 and 301-325). Then we have repeated the fit along the whole spatial axis and used the resulting intensities and widths to scale the sky lines close to the $\mathrm{H} \alpha$ emission.

A similar method was used to subtract the sky lines close to [N II] but in this case, instead of fitting Gaussians, we simply took the mean values along the spatial regions free of [N II] line emission. This method gives much better results than the direct subtraction of the sky exposures (compare the bottom panels in Fig. 2). We also successfully applied this method in the spectra along the minor axis where the sky lines are isolated from the $\mathrm{H} \alpha$ and [N II] emission.

\section{Large-scale dynamics}

The main aim of this study is to investigate the presence of a lagging halo of ionised gas in NGC 2403. This is motivated by our previous H I studies which revealed halos of neutral gas (about 1 tenth of the total $\mathrm{HI}$ ) rotating more slowly than the disk. The halo gas shows up in position-velocity $(p-v)$ diagrams along the major axis of the galaxy as a tail extending towards the systemic velocity (Fraternali et al. 2001).

\subsection{The optical rotation curve}

The optical rotation curves, in $\mathrm{H} \alpha$ and other emission lines, are obtained from the Gaussian fits of the line profiles along the major axis described in Sect. 3.2. Figure 3 shows the $\mathrm{H} \alpha$ rotation curve (the [N II] and [S II] curves are very similar). The left panel shows the rotation velocities for the receding and approaching sides, the right panel shows the rotation curve for the whole galaxy. In the right plot, the errors were estimated from the difference in velocity between the approaching and receding sides. The continuous line shows the H I rotation curve from Fraternali et al. (2002a). Clearly, the ionised and the neutral gas have the same kinematics. The agreement between the $\mathrm{HI}$ and $\mathrm{H} \alpha$ results is remarkable if we consider that the two rotation curves are obtained with different methods and from data with different resolutions. There are systematic 

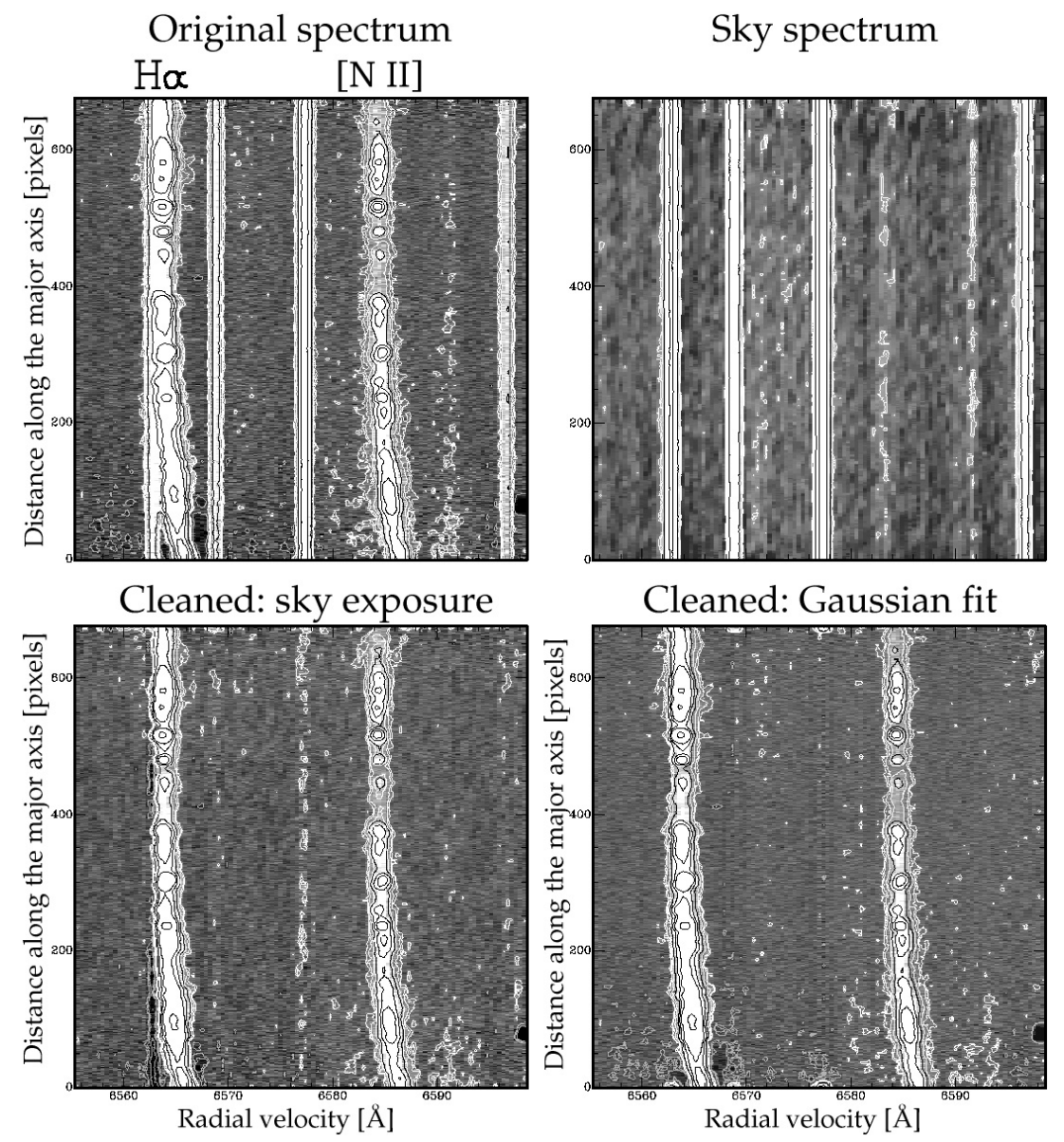

Fig. 2. Optical spectra for NGC 2403 in the spectral range 6555-6600 ̊. The upper left panel shows the original spectrum taken along the major axis of NGC 2403 in the N-W approaching side of the galaxy (slit 1 in Fig. 1), the centre of the galaxy is at the bottom. The curvature of the emission lines ( $\mathrm{H} \alpha$ and $[\mathrm{N} \mathrm{II}])$ is caused by the differential rotation. The upper right panel shows a sky exposure in the same spectral range; a spatial median filter was applied to this image. The lower panels show the same spectrum as the upper left panel after the sky subtraction. Bottom left: sky subtraction obtained using the sky exposure. Bottom right: sky subtraction with the Gaussian fit of the sky lines as described in the text. The second method gives a better result. The negative values in the bottom part of the spectra are probably caused both by stellar light absorption within NGC 2403 and uncertainties in the continuum subtraction. The contours of all these spectra are $3,6,12 \ldots$ in units of rms noise of the original map, $1 \sigma=7.96 \times 10^{-19} \mathrm{erg} \mathrm{s}^{-1} \mathrm{~cm}^{-2} \AA^{-1}$ pixel $^{-1}$.
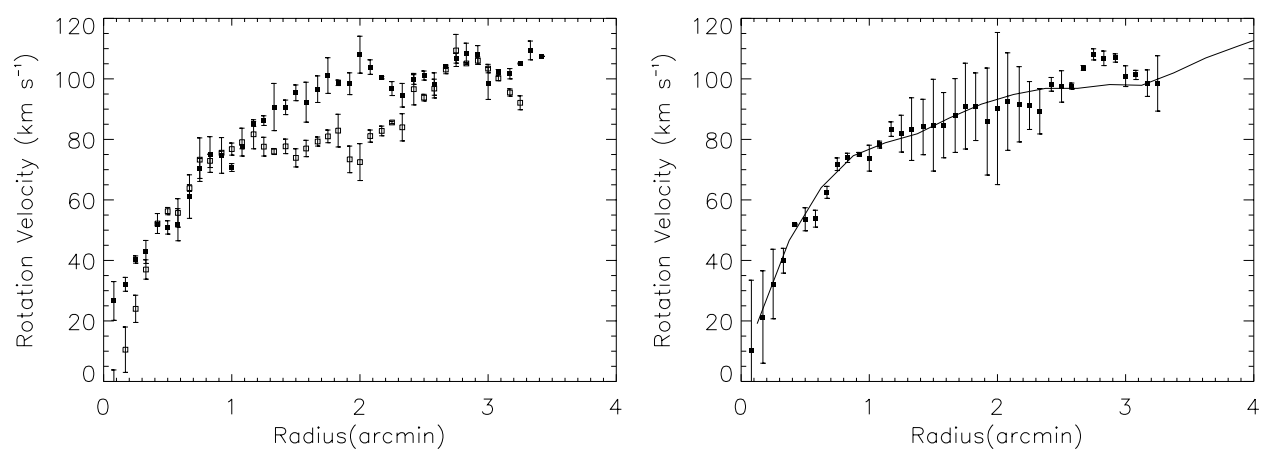

Fig. 3. Optical H $\alpha$ rotation curve for NGC 2403. The left panel shows the rotation velocity for the receding (open squares) and approaching (filled squares) sides of the galaxy. The right panel shows the final rotation curve. In the right plot the error bars are obtained from differences between the approaching and the receding sides and the continuous line shows the H I rotation curve.

differences $\left(\leq 20 \mathrm{~km} \mathrm{~s}^{-1}\right)$ between the approaching a receding rotation curves in the region $1^{\prime}-2.5^{\prime}$, also found in H I. These results from the $\mathrm{H} \alpha$ observations confirm the overall regularity of the disk kinematics of NGC 2403 already shown by the H I study.

\subsection{Lagging ionised gas}

Figure 4 shows the comparison between the $\mathrm{H} \alpha$ and H I position-velocity diagrams (on the same scale) along the major axis of NGC 2403. Both are displayed at full spatial and 


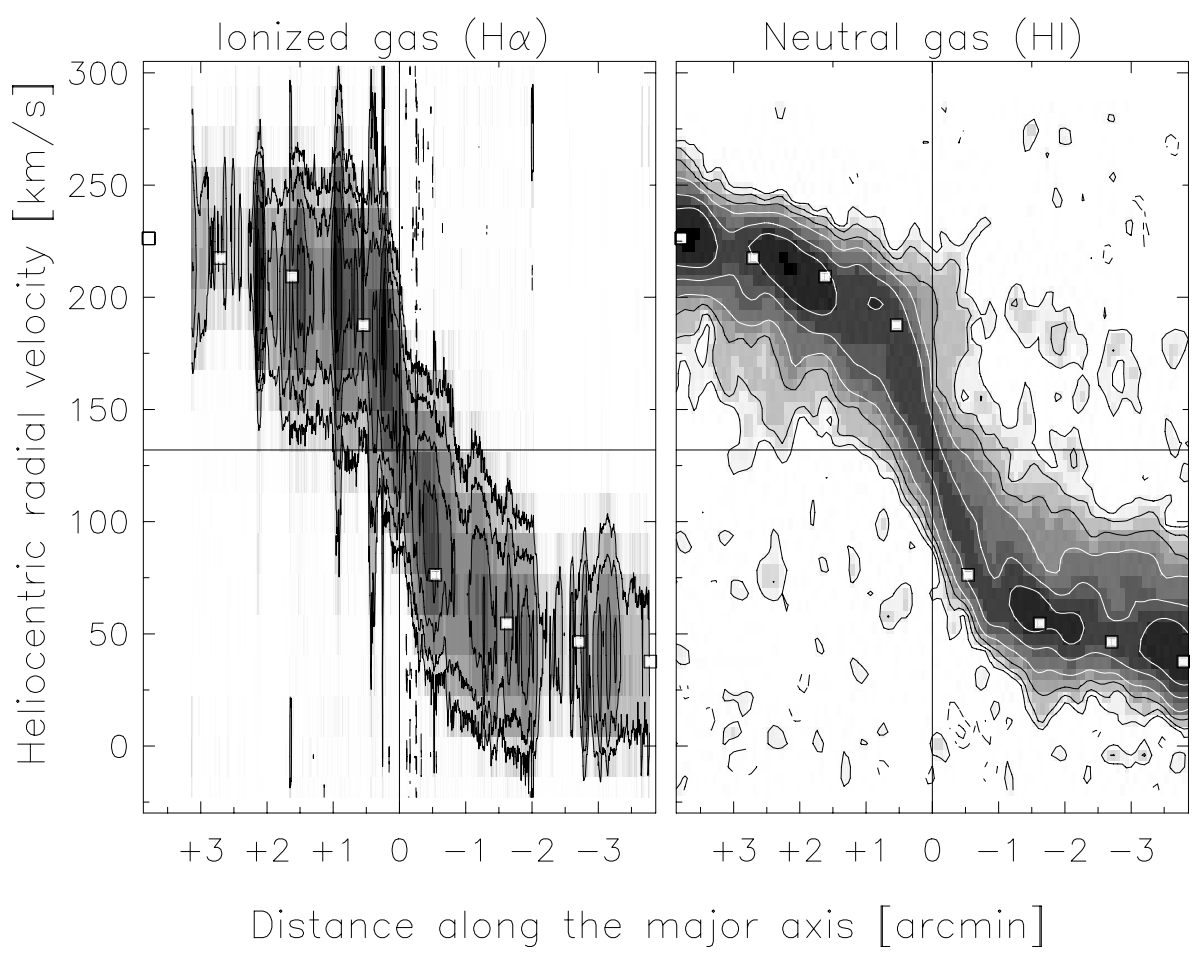

Fig. 4. Position-velocity diagram along the major axis of NGC 2403 for $\mathrm{H} \alpha$ (left) and H I (right), both at full spatial and velocity resolution: $1^{\prime \prime} \times 40 \mathrm{~km} \mathrm{~s}^{-1}$ and $15^{\prime \prime} \times 10 \mathrm{~km} \mathrm{~s}^{-1}$ respectively. The $\mathrm{H} \alpha$ data are obtained by combining the observations along the major axis (slit 1,3 , and 5). The HI data are taken from Fraternali et al. (2002a). The white dots show the H I rotation curve. The contour levels, respectively for $\mathrm{H} \alpha$ and $\mathrm{HI}$ are $-30,-9,-3,3,9,30,75,225,450$ and $-4,-2,2,4,8,16,40,80$ in terms of rms noise.

velocity resolutions: $1^{\prime \prime} \times 40 \mathrm{~km} \mathrm{~s}^{-1}$ and $15^{\prime \prime} \times 10 \mathrm{~km} \mathrm{~s}^{-1}$ respectively for $\mathrm{H} \alpha$ and $\mathrm{HI}$ data. The HI data shown here are taken from Fraternali et al. (2002a). The $\mathrm{H} \alpha$ diagram was obtained by adding (without weighted averaging) all the slit spectra taken along the major axis of the galaxy (see Table 1). The resulting exposure time along the major axis is larger (about a factor 2$)$ in the central $\left(R<1.8^{\prime}\right)$ parts. This causes the decrease of emission beyond $R \sim 1.8^{\prime}$. The white dots are the HI projected rotation curve showing a good agreement between the overall kinematics of the neutral and ionised gas. The central parts of this plot show negative values due to absorption within NGC 2403 and uncertainties in continuum subtraction.

The HI $p$ - $v$ diagram in Fig. 4 (right panel) shows tails of emission towards the systemic velocity (horizontal line). As mentioned above, such tails are the signature of extraplanar gas rotating more slowly than the gas in the disk. Is there a similar component also for the ionised gas? At a first inspection of the $\mathrm{H} \alpha p-v$ diagram in Fig. 4 such a pattern is not clearly visible. This may be partially due to the different resolutions of the ionised and neutral gas data. Figure 5 indeed shows the same $p$ $v$ diagram as in Fig. 4 for $\mathrm{H} \alpha$ (left) and $\mathrm{H} \mathrm{I}$ (right) smoothed at similar angular and velocity resolutions $\left(15^{\prime \prime} \times 40 \mathrm{~km} \mathrm{~s}^{-1}\right)$. In this figure the $\mathrm{HI}$ tails are less visible than in Figure 4 because of the lower velocity resolution. Instead, the ionised gas (left panel) now shows some hints for these tails due to the higher signal-to-noise ratio obtained with the spatial smoothing.

In order to further increase the signal-to-noise ratio, we integrated all the line profiles in the direction along the major axis. Before doing this, we had to remove the overall rotation of the galaxy. To this purpose, we fitted Gaussian functions to each profile using the un-smoothed original data. The fitted intensities were used to normalize the profiles in flux while the fitted velocities were used to shift them in velocity. This method was separately applied to the receding and the approaching sides of the galaxy for each emission line.

The profiles resulting from this integration show that low rotation velocity tails are present for the ionised gas. The composite $\mathrm{H} \alpha$ line profiles for the receding and the approaching sides of the galaxy are shown in Fig. 6 overlaid by a Gaussian fit. The scale is compressed to better show the tails of emission. The panels on the right show the residuals. The velocity in the $x$-axis is rotation velocity in a co-rotating frame (rotation of the disk set to zero). Figure 7 shows the same for the $[\mathrm{N} \mathrm{II}]$ line (faint tails are also detected in [S II] lines, not shown here). From these figures it is clear that the optical line profiles do have tails of emission at low rotation velocities on both sides of the galaxy.

Are these tails the indication of a lagging halo of ionised gas similar to the $\mathrm{HI}$ halo? The ionised tails appear weaker than those observed in $\mathrm{HI}$ line profiles. From the residuals displayed in Figs. 6 and 7 the ionised tail has a brightness of less than 2\% of the line peak, while the typical ratio in the HI profiles is about 10\% (Fraternali et al. 2002a).

The stellar absorption line at $\mathrm{H} \alpha$ can affect the shape of $\mathrm{H} \alpha$ emission line. Using the results obtained by Hoopes \& Walterbos (2003) who have estimated the effect of stellar absorption lines on the gas emission lines, we estimate this to affect the emission from the tails by less than $10-20 \%$. 


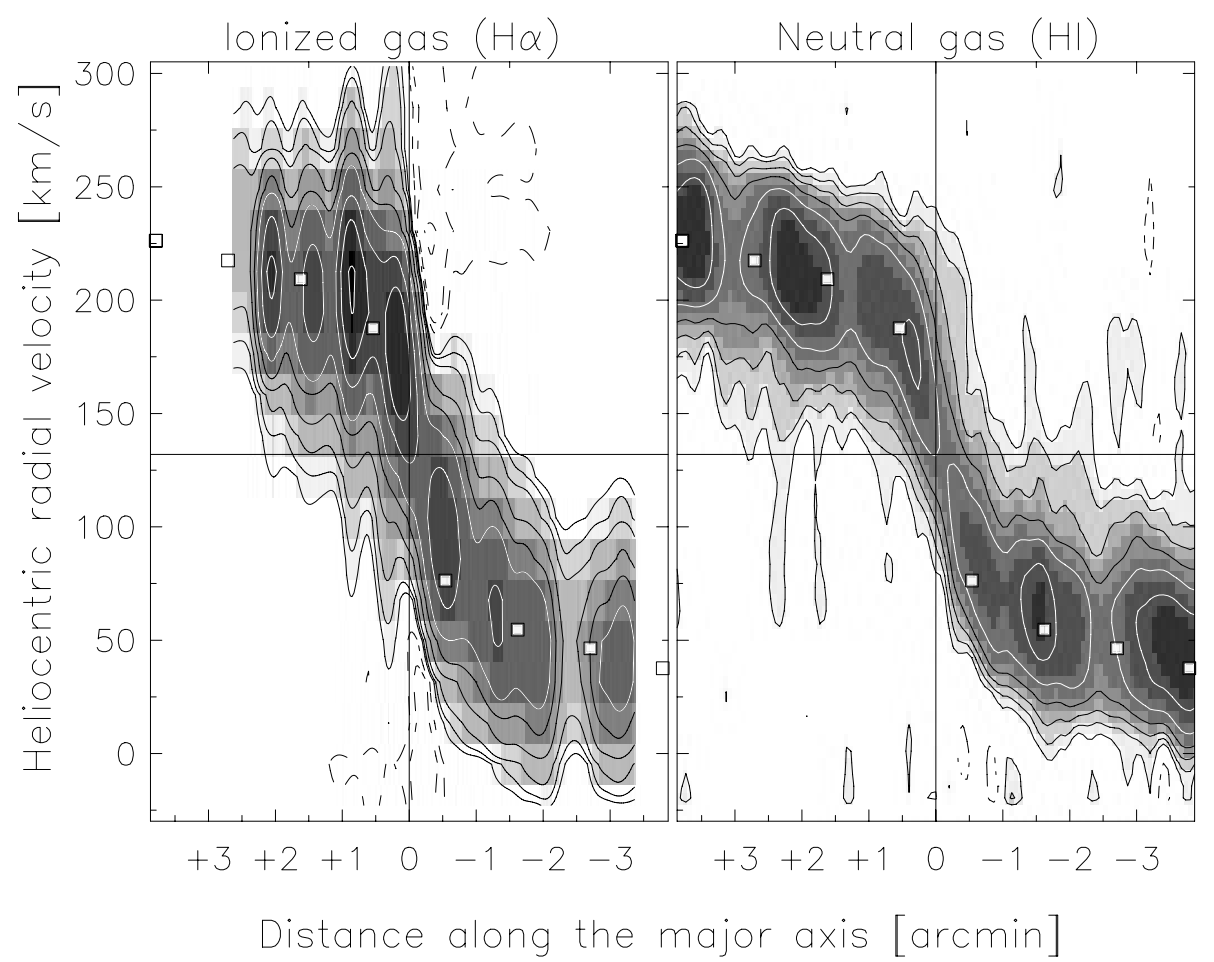

Fig. 5. Position-velocity diagrams along the major axis of NGC 2403 for $\mathrm{H} \alpha$ (left panel) and H I (right panel) at similar spatial and velocity resolutions. The dots show the H I rotation curve. The contour levels, respectively for $\mathrm{H} \alpha$ and H I are $-12,-6,-3,3,6,12,30,66,120,300$, 600,1200 and $-4,-2,2,4,8,16,32,64$ in terms of rms noise of the smoothed data. The negative levels in the central regions of the H $\alpha$ plot are due to continuum subtraction and stellar absorption in NGC 2403.

Moreover, the fact that we obtain the same results from both the $\mathrm{H} \alpha$ and the [N II] line indicates that the effect is small.

For a proper comparison between the lagging neutral and ionised gas, one should not consider the ratio in emission but the mass ratio. The HI emission is linearly related to the mass leading to a ratio between the mass of the cold $\mathrm{HI}$ disk and the halo gas of about 10\% (Fraternali et al. 2002a; Schaap et al. 2000). Instead, for the ionised gas such a relation is rather complex. Considering two scale heights for the thin disk and the lagging gas $h_{1}$ and $h_{2}$ respectively, a $2 \%$ ratio in emission gives a $14 \frac{h_{1}}{h_{2}} \%$ ratio in masses (assuming the same filling factor for the two components). Finally, the mass of the lagging ionised gas is probably under-estimated also because of the poor velocity resolution of the optical spectra that partly hides the lagging gas from detection.

Concerning the kinematics, the ionised and neutral components seem to match quite closely. The tails of ionised gas are located (as those of $\mathrm{HI}$ ) only at low rotation velocities on both sides of the galaxy. The mean rotation velocity of the ionised lagging gas (about $80 \mathrm{~km} \mathrm{~s}^{-1}$ lower than the rotation in the disk) is somewhat larger than that found in H I but this result is strongly affected by the poor spectral resolution. The ionised tails seem to extend up to more than $100 \mathrm{~km} \mathrm{~s}^{-1}$, almost down to the systemic velocity of NGC 2403, and such an extension is very similar to the extension of the tails of neutral gas in the central regions of the galaxy. In some positions, the tails of ionised gas extend far beyond the systemic velocity of the galaxy (see Sect. 4.1) as also found for some high velocity H I complexes (Fig. 4).
Finally, we have investigated the line ratios between different emission lines. Halo gas usually shows a higher [N II] $/ \mathrm{H} \alpha$ ratio with respect to gas in the disk (e.g., Hoopes et al. 1999). Figure 8 shows a plot of this ratio versus rotation velocity (rotation of the disk is set to zero). This plot was obtained from the composite profiles shown in Figs. 6 and 7 , therefore the ratio is normalized at zero velocity. The $[\mathrm{N} \mathrm{II}] / \mathrm{H} \alpha$ ratio rises for gas at lower rotation velocity. This clearly strengthens the interpretation that such a gas is located above the plane and also suggests that the higher the scaleheight of the gas, the lower is its rotation velocity in accordance with results obtained for edge-on galaxies (e.g., Rand 2000).

In conclusion, it appears that the tails at low rotation velocity in optical emission lines found in NGC 2403 represent a lagging component of ionised gas which seems like the counterpart of the lagging $\mathrm{HI}$ halo. This is the first time that a lagging halo is detected in ionised gas in a non edge-on galaxy. The scale-height of such a component is unknown because of the inclination of the galaxy. However, an analogy with the extended and lagging DIG layers of edge-on galaxies such as NGC 5775 (Rand 2000) and NGC 891 (Rand 1997) suggests scale-heights of a few kpc.

\subsection{Large-scale non-circular motions}

In Sect. 3.2 we have presented the evidence for a halo component of ionised gas with lower rotation velocity with respect to the gas in the disk. Here we start investigating the presence of non-circular motions. In this section we concentrate on 

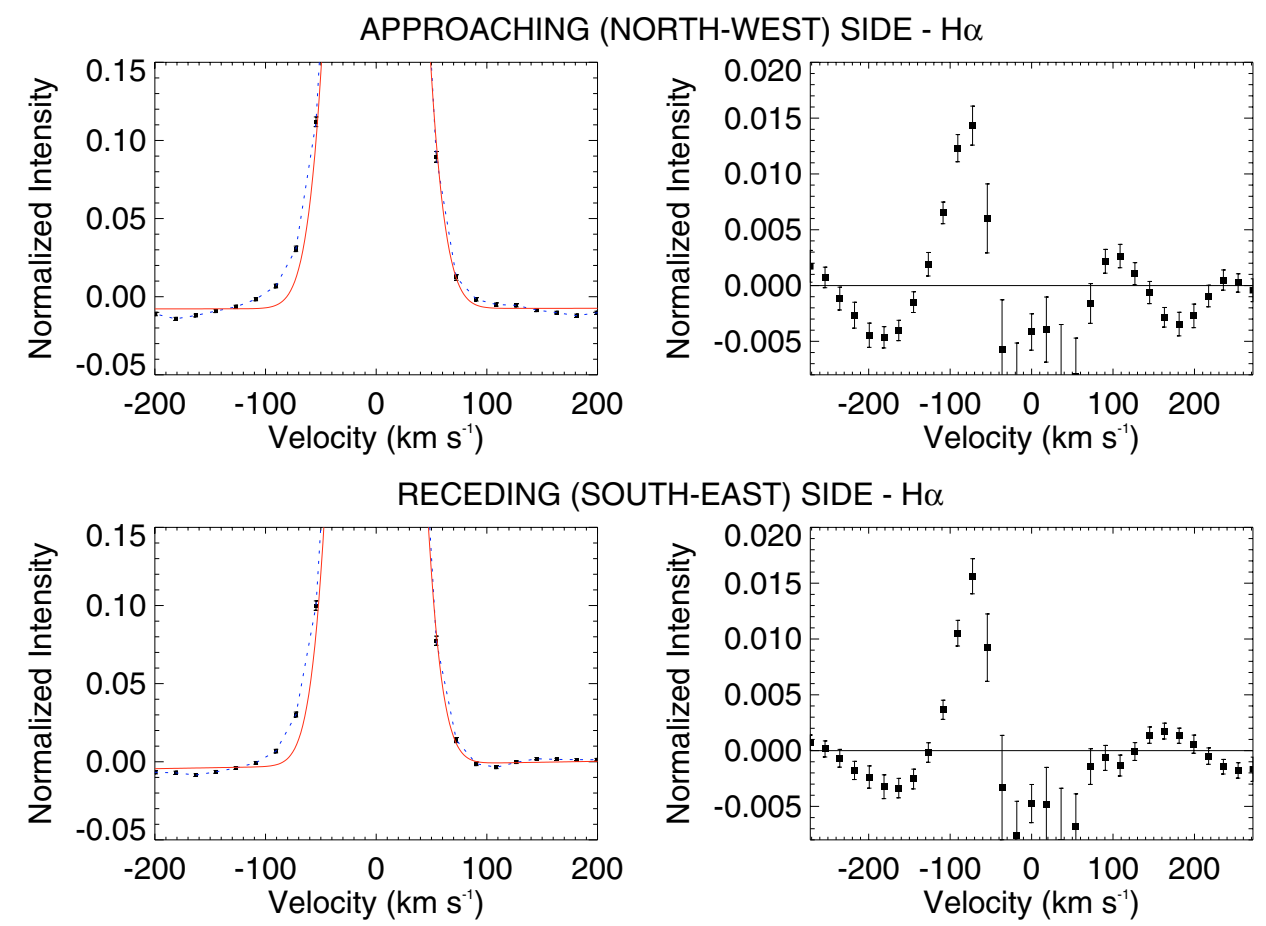

Fig. 6. Composite $\mathrm{H} \alpha$ line profiles along the major axis of NGC 2403 for both the approaching (upper panels) and receding (lower panels) sides of NGC 2403. The continuous line indicates the Gaussian fit. The tails are visible at low rotation velocities (negative velocities in these plots). Right panels show the residuals.
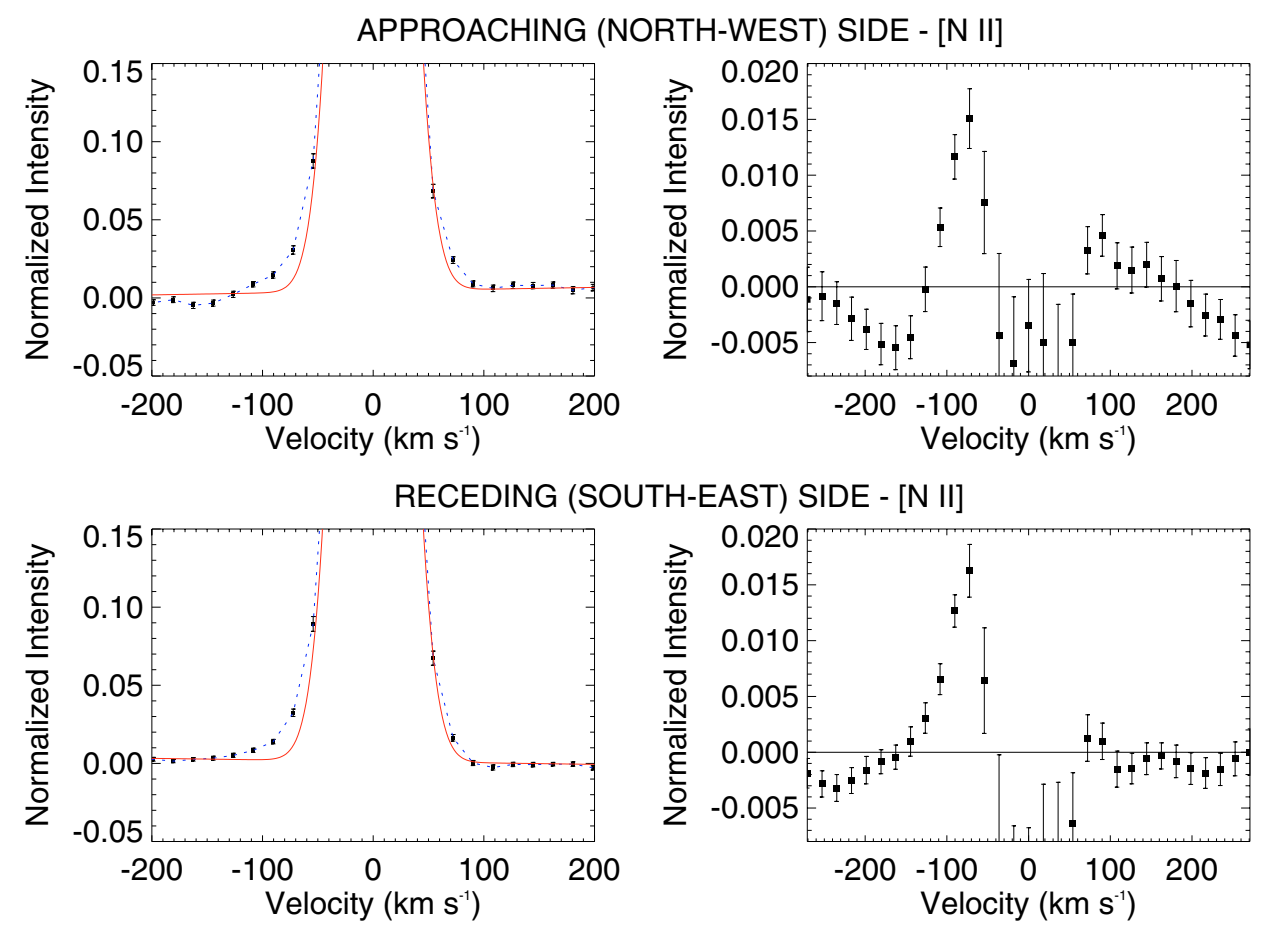

Fig. 7. Composite [N II] line profiles along the major axis of NGC 2403 for both the approaching (upper panel) and receding (lower panel) sides of NGC 2403. The continuous line indicates the Gaussian fit. The tails are visible at lower rotation velocities (negative velocities in these plots). Right panels show the residuals.

large-scale radial motions of the ionised gas. In Sect. 4.1 we discuss local features showing substantial vertical motions.

Radial motions are revealed by $p-v$ diagrams along and parallel to the minor axis, where they produce an asymmetry between the near and the far side of the galaxy
(Fraternali et al. 2001). Indeed, the minor axis is the locus where there is no contribution by the rotational velocity and where non-circular motions would show up.

In order to look for such effects in the ionised component we have used the slits along the minor axis of NGC 2403 


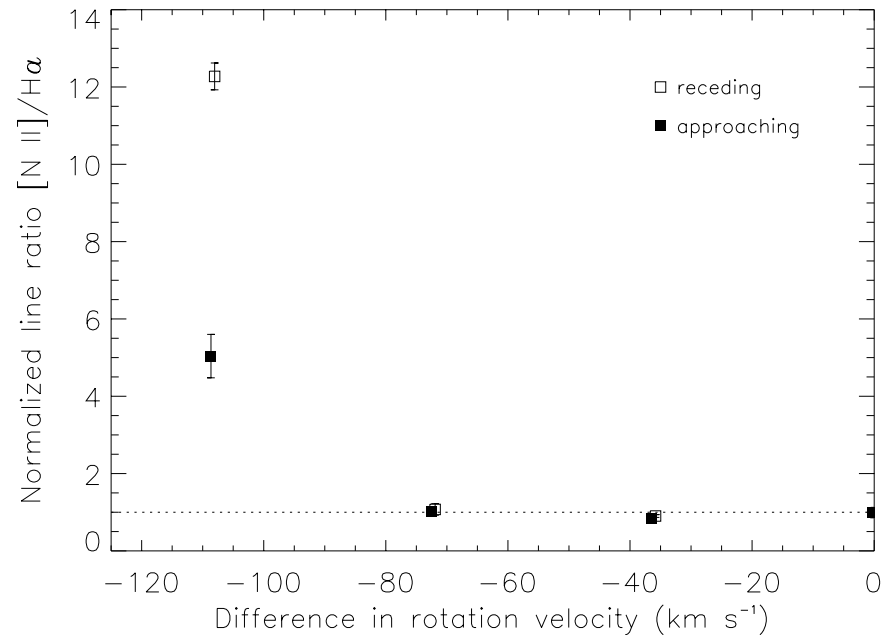

Fig. 8. $[\mathrm{N} \mathrm{II}] / \mathrm{H} \alpha$ intensity ratio versus velocity difference from the rotation of the disk. The ratio is normalized at zero velocity. The line ratio shows an increase at decreasing rotation velocities as expected if this gas is located above the plane of the disk.

(slits 2, 4, and 6, see Fig. 1). We have searched for tails of emission for the ionised gas using Gaussian fits as done for the major axis (Sect. 3.2). Figure 9 shows the composite $\mathrm{H} \alpha$ line profiles in both sides of the galaxy. The near side of the galaxy is the South-West side for the spiral arms to be trailing and also considering dust absorption features on the optical image. Faint tails of emission are detected in particular in the South-West (near) side of the galaxy, at velocities higher than systemic. In the North-East (far) side the tail is much fainter. However, this side has the lowest total exposure time $(1.5 \mathrm{~h}$ in the outer and 4.5 in the inner part, see Table 1).

If one interprets these two $\mathrm{H} \alpha$ tails in terms of large-scale non-circular motion, the ionised gas (as the $\mathrm{HI}$ ) would have a radial inflow towards the galactic centre. Unfortunately, these tails are very faint (at least two times fainter that those along the major axis) and a firm conclusion from this analysis is not possible. No additional information comes from the study of [N II] and [S II] lines.

The kinematic position angle of NGC 2403 is known with a precision of 1-2 degrees from $\mathrm{H}$ I observations and we exclude that such tails could have been produced by a misalignment between the slits and the minor axis. This has also been tested by building models of galactic disks with different orientation of the minor axis. The HI velocity field of NGC 2403 is regular and shows no sign of wiggles between arm and inter-arm regions (Fraternali et al. 2001). Moreover, the harmonic analysis of the H I velocity field (Schoenmakers et al. 1997) shows that the disk of NGC 2403 is axisymmetric to a fairly high degree. Therefore we exclude that the tails of ionised gas at anomalous velocity are produced by integrating through regions of the disk with different kinematics. The possibility that the gas in the tail and the bulk of the gas spatially co-exists, with such a different kinematics, is quite unlikely, these being collisional systems.

If these tails are indeed real and part of a large-scale radial inflow of ionised gas, this could be a fraction of the inflowing neutral gas which is being ionised by the stellar continuum in the central parts of the galaxy. As for the neutral gas, the presence of inflow motion may indicate that we are observing the last stage of the fountain when the gas is falling back to the disk and moving inwards (Bregman 1980). However, as already concluded for H I (Fraternali et al. 2001), the same pattern would occur if the gas originates from accretion from the intergalactic medium. Therefore, the possible detection of large-scale radial inflow of ionised gas does not confirm nor exclude the presence of a fountain.

On the other hand, if a galactic fountain is at work, we would expect to observe an outflow of ionised gas tracing the initial stage of the process. Here we have shown that such outflow is not detected as a large-scale radial motion. However, on the small scales, there is evidence for significant outflow (vertical motions) of gas from the disk of NGC 2403. This is discussed in Sect. 4.1.

\section{Small-scale structures}

\subsection{Outflows of ionised gas}

Figure 10 (left panel) shows a spectrum along the major axis (slit 5) of NGC 2403 in the spectral range of $\mathrm{H} \alpha$ and [N II]. At some locations, this spectrum shows a striking broadening of the profiles visible in each line. They are labeled 2 and 3 and are at, respectively, about $0.5^{\prime}$ and $1^{\prime}$ from the centre of the galaxy (arrow). The very broad feature detected only in $\mathrm{H} \alpha$ and labeled 1 is discussed in Sect. 4.2. The bottom right panel shows the $\mathrm{H} \alpha$ line profile taken along feature 2. This profile shows long tails on both sides, at higher and lower velocity with respect to the rotation (peak of the profile). On the side of low velocities, the tail is much more pronounced and extends $200 \mathrm{~km} \mathrm{~s}^{-1}$ away from rotation. On the high velocity side it reaches $300 \mathrm{~km} \mathrm{~s}^{-1}$ heliocentric velocity.

Both features 2 and 3 are spatially resolved. Their physical sizes are about 50-100 pc. In the $\mathrm{H} \alpha$ image (Fig. 1) they correspond to compact $\mathrm{H}$ II regions of medium brightness. There are no clear counterparts in $\mathrm{HI}$ and no X-ray counterparts are seen in the Chandra image of NGC 2403 (Fraternali et al. 2002b; Schlegel \& Pannuti 2003). The probable explanation of such local broadening of the line profiles is that of strong vertical motions in regions of high star formation activity. We searched for relations with known supernova remnants and found a possible association (within our positional errors) with two SNRs for the feature 2 (numbers 14 and 15 in the catalogue of Matonick et al. 1997) and no association for feature 3.

The presence of such features clearly points at outflows of gas from the disk of NGC 2403. This can be part of the early stage of an ongoing galactic fountain. The question is whether or not this process can be powerful enough to account for all the extraplanar gas. To answer it, we have estimated the flux density of the high velocity gas in such features. Consider first feature 2. If we integrate only the emission at projected velocity differences from rotation of more than $50 \mathrm{~km} \mathrm{~s}^{-1}$ we obtain an $\mathrm{H} \alpha$ flux of $2 \times 10^{-15} \mathrm{erg} \mathrm{s}^{-1} \mathrm{~cm}^{-2}$ (luminosity of $2.4 \times 10^{36} \mathrm{erg} \mathrm{s}^{-1}$ ) and an emission measure $(E M)$ of about $175 \mathrm{pc} \mathrm{cm}^{-6}$, assuming a gas temperature of $10^{4} \mathrm{~K}$. The emission measure $E M$ is related to the electron density $n_{\mathrm{e}} E M\left(\mathrm{pc} \mathrm{cm}^{-6}\right)=\int n_{\mathrm{e}}^{2} \mathrm{~d} l$ (Spitzer 1978), this leads 

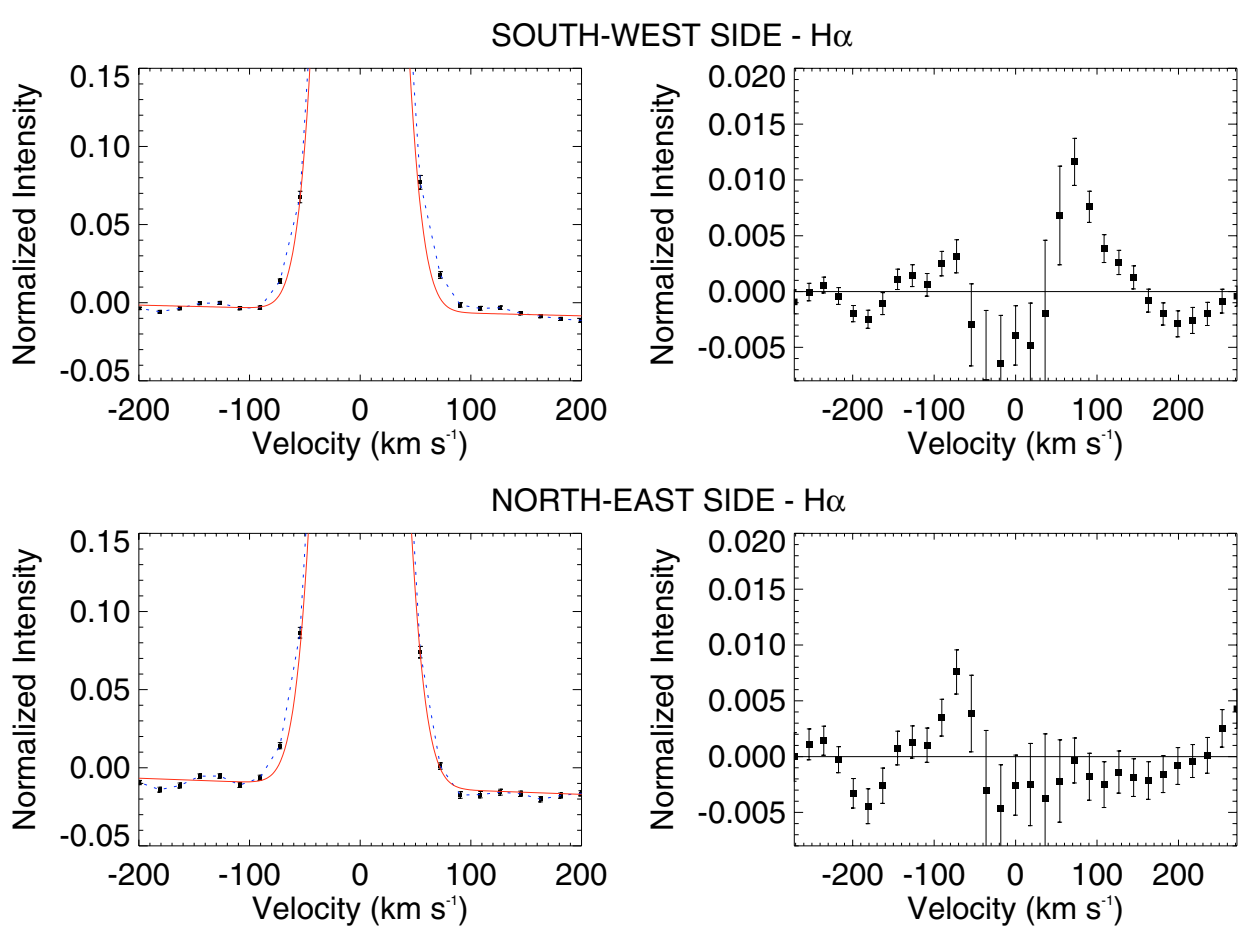

Fig. 9. Composite line profiles for $\mathrm{H} \alpha$ along the minor axis of NGC 2403 in the S-W and N-E sides of the galaxy (left panels). The continuous line indicates the Gaussian fit. The right panels show the residuals. Velocities are with respect to systemic. The tail at high velocity in the S-W (near) side and at low velocity in the N-E (far) side may indicate a large-scale radial inflow.
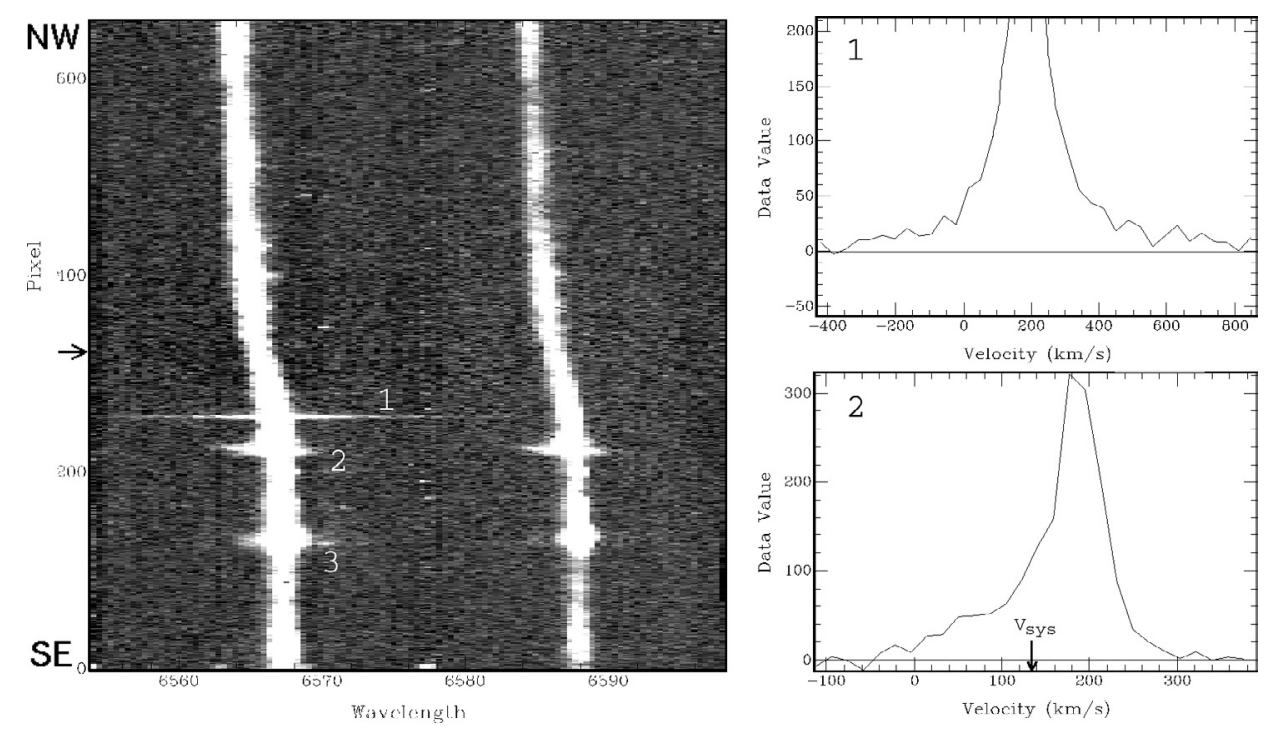

Fig. 10. Left panel: optical spectrum along the major axis of NGC 2403 (slit 5 in Fig. 1) in the spectral range of H $\alpha$ and [N II]. Note the large velocity dispersion of features visible in the receding (S-E) side of the galaxy (bottom of this image). Right panels: H $\alpha$ line profiles through the broad features 1 and 2 observed along the major axis of NGC 2403 and shown in the left panel. The velocities are heliocentric.

to an rms electron density for feature 2 of $\left\langle n_{\mathrm{e}}\right\rangle_{\mathrm{rms}}=1.6 \mathrm{~cm}^{-3}$, assuming a size of $70 \mathrm{pc}$ for the region of emission.

The filling factor is defined as $f=\left(\frac{\left\langle n_{\mathrm{e}}\right\rangle_{\mathrm{rms}}}{n_{\mathrm{e}}}\right)^{2}$ where $n_{\mathrm{e}}$ is the electron density of the ISM as inferred using the ratio of emitted intensities of forbidden doublets. We used the ratio of $\left[\mathrm{S}_{\mathrm{II}}\right]$ lines and found a value of $\frac{J 6716}{J 6731}=1.42 \pm 0.02$. Unfortunately this does not help us to clearly constrain $n_{\mathrm{e}}$ and values between 10 and $100 \mathrm{~cm}^{-3}$ (for $T=10^{4} \mathrm{~K}$ ) are all acceptable (Osterbrock 1974). We thus obtain a filling factor for the high velocity gas in the feature 2 in the range $f=20-0.2 \times 10^{-3}$. Finally, assuming a spherical shape for the region of emission, we get a value for the $\mathrm{H}^{+}$mass of $3.1 \times\left(\frac{f}{0.002}\right)^{1 / 2} 10^{2} M_{\odot}$ where $f$ is the filling factor. Such value for the mass correspond to a kinetic energy of about $4.5 \times\left(\frac{f}{0.002}\right)^{1 / 2} \times 10^{49} \mathrm{erg} \mathrm{s}^{-1}$. 
For feature 3 we find an $E M=410 \mathrm{pc} \mathrm{cm}^{-6}$, an rms electron density of $\left\langle n_{\mathrm{e}}\right\rangle_{\mathrm{rms}}=2.3 \mathrm{~cm}^{-3}$ and a slightly higher filling factor $f \simeq 70-0.7 \times 10^{-3}$. The mass estimate in this case is $1.3 \times\left(\frac{f}{0.007}\right)^{1 / 2} 10^{3} \quad M_{\odot}$ and the kinetic energy $4.7 \times\left(\frac{f}{0.007}\right)^{1 / 2} \times 10^{49} \mathrm{erg} \mathrm{s}^{-1}$.

From our H I observations, which indicate an $\mathrm{H} \mathrm{I}$ inflow velocity of about $15 \mathrm{~km} \mathrm{~s}^{-1}$, we estimate an infall rate $\dot{M}_{\mathrm{HI}} \sim$ $0.9 \times\left(\frac{L}{5 \mathrm{kpc}}\right) M_{\odot} \mathrm{yr}^{-1}$, where $L$ is the scale length of the process (Fraternali et al. 2002a). Assuming a continuous cycle (i.e. assuming the same scale length) between ionised and neutral gas, the contribution to the outflow rate of ionised gas from features like the ones described above would be $\dot{M}_{\mathrm{H}+} \approx$ $0.5-2.0 \times\left(\frac{L}{5 \mathrm{kpc}}\right) \times 10^{-5} M_{\odot} \mathrm{yr}^{-1}$ with filling factors in the range $f=1-10 \times 10^{-3}$. Therefore one would need tens of thousands of such features to match the observed inflow of H I gas. Note that this latter value does not depend on the chosen scale length but only on the ratio between the mass and the velocity of the out/in-flowing gas. With the available data, it is hard to say whether this can be the case in NGC 2403. With our slits we have sampled a very small fraction of the disk of NGC $2403(\leq 1 \%)$. Moreover, none of our slits intercepts very bright $\mathrm{H}$ II regions which can easily be hundreds times more efficient than the two detected here. Finally, part of the outflowing gas can also be hotter than the ionised gas observed in recombination lines (Fraternali et al. 2002b).

To sum up, we have detected local vertical outflows of ionised gas that possibly feed a galactic fountain process in NGC 2403. Such a process could be responsible for the origin of the halo gas if the galaxy hosts several thousands outflow regions like the two reported here.

\subsection{Stellar outflow or Supernova?}

The extremely broad $\mathrm{H} \alpha$ emission feature shown in Fig. 10 (labeled 1) was detected in two exposures (observation 6 in Table 1) in the third night of the observations. Other two exposures along the major axis (observation 7), which were obtained in the same night with a small shift of less than 2 "NorthEast, did not show any broadening in that position while the other features were still present and had similar shapes and locations. Clearly, in contrast with the others, feature 1 is unresolved (size smaller than $10-15 \mathrm{pc}$ ) and, therefore, probably stellar. It is located at about $300 \mathrm{pc}$ from the centre of the galaxy and has a total velocity extent of about $1200 \mathrm{~km} \mathrm{~s}^{-1}$ (see the line profile in the upper right panel of Fig. 10). Its total $\mathrm{H} \alpha$ flux (only the wings) is about $1.2 \times 10^{-15} \mathrm{erg} \mathrm{s}^{-1} \mathrm{~cm}^{-2}$ (luminosity of $1.4 \times 10^{36} \mathrm{erg} \mathrm{s}^{-1}$ ). It is to far to be connected with the nucleus of NGC 2403; moreover NGC 2403 does not show any evidence of nuclear activity. No Chandra X-ray source is found at the position of this feature (Fraternali et al. 2002b; Schlegel \& Pannuti 2003).

The most interesting characteristic of this broad feature is that the emission is clearly visible in $\mathrm{H} \alpha$ but not in any other line, except for a weak signal in $\mathrm{H} \beta$ in the blue arm of the ISIS spectrograph. Velocities of a few thousands $\mathrm{km} \mathrm{s}^{-1}$ are indeed observed for ejected materials from Wolf Rayet stars (Crowther 2000) or Blue Luminous
Variables (Stahl et al. 2001). However, in such stars also helium (and [ $\left.\begin{array}{ll}\mathrm{N} & \mathrm{II}\end{array}\right]$ ) lines, absent here, are usually detected. Another possibility is that of emission from a supernova envelop (Chu \& Kennicutt 1988). The velocity of about $500-1000 \mathrm{~km} \mathrm{~s}^{-1}$ is typical for the Sedov phase in the expansion of a supernova envelop and SN age less than $10^{4} \mathrm{yrs}$. A supernova rate of $0.01 \mathrm{yr}^{-1}$ for NGC 2403 (Matonick et al. 1997) would mean about 100 detectable SNs in the entire disk, consistent with our result, considering that our observations sample only about $1 \%$ percent of the disk. However, also in the case of a supernova the absence of other emission lines (e.g. [S II]) could present a problem.

\section{Conclusions}

We have discussed optical long-slit spectroscopy of the spiral galaxy NGC 2403. The purpose of these observations was to study the kinematics of the ionised gas and to compare it with the H I results by Fraternali et al. (2001, 2002a) which show the presence of an extended $\mathrm{H}$ I halo rotating more slowly (lagging) with respect to the disk.

The results from this optical study of NGC 2403 can be summarized as follows:

1. The overall kinematics of neutral and ionised gas as inferred from the HI line and optical recombination lines ( $\mathrm{H} \alpha$ and $[\mathrm{N} \mathrm{II]})$ agree remarkably well. The $\mathrm{HI}$ and $\mathrm{H} \alpha$ rotation curves agree also in the very inner regions of the galaxy.

2. The slit spectra along the major axis show tails of emission at velocities lower than rotation (towards systemic). Such tails are produced by a large-scale lagging component of ionised gas which appears to be the ionised counterpart of the lagging $\mathrm{H}$ I halo. This interpretation is confirmed by the presence of a gradient in the $[\mathrm{N} \mathrm{II}] / \mathrm{H} \alpha$ intensity ratio with decreasing rotation velocity. The lagging halo gas has already been observed in edge-on galaxies (DIG) but this is the first detection in a galaxy viewed at intermediate inclination.

3. Slits along the minor axis of the galaxy seem to indicate a large-scale radial inflow of the ionised gas towards the centre of the galaxy (as found for HI). If the present results are correct, this would imply that the DIG does not represent the outflowing component of the fountain. However, because of the faintness of the ionised tails and the poor velocity resolution, a firm conclusion is not possible.

4. On small scales, there is clear evidence for powerful vertical outflows of ionised gas. Such outflows are detected as local broadenings of the line profiles of up to $300 \mathrm{~km} \mathrm{~s}^{-1}$. Such broad features are probably produced by large vertical motions and originate in regions of ongoing star formation like compact H II regions. In our observations we have detected two such features, but we have sampled only a tiny fraction $(\leq 1 \%)$ of the galactic disk. In the hypothesis of a galactic fountain, in order to match the observed $\mathrm{HI}$ infall rate one would need tens of thousands of such outflows over the all disk. 
5. Also, there are implications for the study of our Galaxy. As already pointed out by Fraternali et al. (2001, 2002a), the halo $\mathrm{H}$ I features found in external galaxies are probably the analogue of some of the galactic HVCs. Recently, ionised counterparts of several HVCs and also ionised HVCs with no neutral gas have been found (Richter et al. 2003). The present detection of an ionised counterpart of the $\mathrm{HI}$ halo in NGC 2403 showing a kinematics very similar to that of the HI, strengthens this analogy with the galactic HVCs.

Acknowledgements. We are grateful to an anonymous referee for various important improvements to the paper.

\section{References}

Bregman, J. N. 1980, ApJ, 236, 577

Collins, J. A., Rand, R. J., Duric, N., \& Walterbos, R. A. M. 2000, ApJ, 536, 645

Crowther, P. A. 2000, A\&A, 356, 191

Chu, Y.-H., \& Kennicutt, R. C., Jr. 1988, AJ, 95, 1111

Donahue, M., Aldering, G., \& Stocke, J. T. 1995, ApJ, 450, L45

Drissen, L., Roy, J.-R., Moffat, A. F. J., \& Shara, M. M. 1999, AJ, 117, 1249

Ferguson, A. M. N., Wise, R. F. G., \& Gallagher, J. S. 1996, AJ, 112, 2567

Fraternali, F., Oosterloo, T., Sancisi, R., \& Van Moorsel, G. 2001, ApJ, $562, \mathrm{~L} 47$

Fraternali, F., Van Moorsel, G., Sancisi, R., \& Oosterloo, T. 2002a, AJ, 123, 3124

Fraternali, F., Cappi, M., Sancisi, R., \& Oosterloo, T. 2002b, ApJ, 578, 109

Fraternali, F., Oosterloo, T., Boomsma, R., Swaters, R., \& Sancisi, R. 2003, Recycling Intergalactic and Interstellar Matter, IAU, Symp., 217,44
Hoopes, C. G., Walterbos, R. A. M., \& Rand, R. J. 1999, ApJ, 522, 669

Hoopes, C. G., \& Walterbos, R. A. M. 2003, ApJ, 586, 902

Karachentsev, I. D., Sharina, M. E., Grebel, E. K., et al. 1999, A\&A, 352,399

Madore, B. F., \& Freedman, W. L. 1991, PASP, 103, 933

Matonick, D. M., Fesen, R. A., Blair, W. P., \& Long, K. S. 1997, ApJS, 113,333

Matthews, L. D., \& Wood, K. 2003, ApJ, 593, 721

Oort, J. H. 1970, A\&A, 7, 381

Osterbrock, D. E. 1974, Astrophysics of gaseous nebulae (San Francisco: Freeman)

Osterbrock, D. E., Fulbright, J. P., Martel, A. R., et al. 1996, PASP, 108,277

Rand, R. J. 1997, ApJ, 474, 129

Rand, R. J. 2000, ApJ, 537, L13

Rand, R. J., Kulkarni, S. R., \& Hester, J. J. 1992, ApJ, 396, 97

Reynolds, R. J. 1991, in The interstellar disk-halo connection in galaxies, ed. H. Bloemen (Dordrecht: Kluwer), IAU Symp., 144, 67

Richter, P., Wakker, B. P., Savage, B. D., \& Sembach, K. R. 2003, ApJ, 586, 230

Schaap, W. E., Sancisi, R., \& Swaters, R. A. 2000, A\&A, 356, L49

Schlegel, E. M., \& Pannuti, T. G. 2003, AJ, 125, 3025

Schoenmakers, R. H. M., Franx, M., \& de Zeeuw, P. T. 1997, MNRAS, 292, 349

Shapiro, P. R., \& Field, G. B. 1976, ApJ, 205, 762

Spitzer, L. 1978, Physical processes in the interstellar medium (New York: Wiley)

Stahl, O., Jankovics, I., Kovács, J., et al. 2001, A\&A, 375, 54

Swaters, R. A., Sancisi, R., \& van der Hulst, J. M. 1997, ApJ, 491, 140

Valdes, F. 1992, in Astronomical data analysis software and systems, ed. D. M. Worrall, C. Biemesderfer, \& J. Barnes (San Francisco: ASP), ASP Conf. Ser., 25, 417

Wakker, B. P., \& van Woerden, H. 1997, ARA\&A, 35, 217 